\title{
Imagerie résolue en temps de plasma créé par décharge capillaire ablative pour l'amplification de rayonnement $X$ mou
}

\author{
J. Pons, R. Dussart, S. Götze, D. Hong, R. Viladrosa, C. Cachoncinlle, C. Fleurier \\ et J.-M. Pouvesle
}

GREMI-ESPEO, UMR 6606 du CNRS, Université d'Orléans, 14 rue d'Issoudun, BP. 6744 , 45067 Orléans cedex 2, France

\begin{abstract}
Résumé. L'évolution temporelle de la répartition spatiale du rayonnement émis lors d'une décharge ablative dans un capillaire de polyéthylène a été mesurée, dans le but d'étudier la faisabilité d'amplification de rayonnement $\mathrm{X}$ mou. Des images résolues en temps du plasma émetteur, dans les domaines spectraux allant du VUV aux X mous, ont été réalisées par le biais d'un système pinhole. Cette étude a permis d'observer les différentes phases de l'évolution du plasma, et d'envisager une future optimisation du système de décharge employé pour l'obtention d'un laser X mou.
\end{abstract}

\section{INTRODUCTION}

Ce travail entre dans le cadre de l'étude d'une décharge capillaire ablative destinée à réaliser un effet d'amplification de la raie Balmer- $\alpha$ émise à $18,2 \mathrm{~nm}$ par l'ion hydrogénoïde $C^{5+}$, via un schéma de pompage par recombinaison à 3 corps. Celui-ci est favorisé par une forte densité électronique, mais une. faible température. Pour obtenir de telles conditions il est nécessaire de réaliser une auto-compression magnétique (Z-pinch) du plasma, suivie d'un refroidissement brutal favorisant la recombinaison de l'ion $\mathrm{C}^{6+}$ et le déséquilibre thermodynamique entre les niveaux de la transition d'intérêt, par expansion et contact thermique avec les parois du capillaire.

Le but des expériences décrites ici est de visualiser l'évolution temporelle du profil radial d'émission du plasma, permettant d'observer une éventuelle auto-compression ainsi que l'interaction plasma-paroi (ablation, contact thermique), en vue d'optimiser le système de décharge pour l'obtention du schéma de pompage.

\section{DISPOSITIF EXPERIMENTAL}

On crée un plasma de carbone et d'hydrogène par ablation de la paroi interne d'un capillaire de polyéthylène, en déchargeant un condensateur d'une capacité de $100 \mathrm{nF}$ dans le canal préionisé du capillaire (initialement sous vide secondaire), d'un diamètre de $1 \mathrm{~mm}$ pour une longueur comprise entre 10 et $16 \mathrm{~mm}$. Ce dispositif permet d'obtenir un courant de décharge de plusieurs dizaines de $\mathrm{kA}$ sur environ $50 \mathrm{~ns}$, et d'injecter dans le capillaire une densité de puissance de plusieurs dizaines de $\mathrm{GW} . \mathrm{cm}^{-3}$. Des expériences de spectroscopie [1] ont mis en évidence la présence de l'ion $\mathrm{C}^{5+}$ au cours de la première demi période du courant et permettent de penser que l'ion $\mathrm{C}^{6+}$ est présent lorsque le courant est maximal.

Le rayonnement émis par le plasma est recueilli sur un détecteur $\mathrm{MCP}$ couplé à une caméra $\mathrm{CCD}$ intensifiée ; ce système permet l'acquisition d'images avec une résolution temporelle d'environ $20 \mathrm{~ns}$. L'image du plasma est formée sur le MCP (grandissement $=6$ ) au travers d'un diaphragme de $50 \mu \mathrm{m}$ de diamètre ("pinhole"). Le schéma détaillé de l'expérience peut être trouvé dans l'article [1].

\section{ETUDE DE LA REPARTITION SPATIALE DE L'EMISSION DU PLASMA}

Le profil radial d'émission du plasma a été observé pour différents stades de la décharge, dans différentes régions spectrales afin de visualiser les différents degrés d'ionisation. La figure suivante montre les 
images pinhole obtenues pour différents instants de la décharge (pointés sur la courbe de courant) avec un capillaire de $10 \mathrm{~mm}$ de longueur soumis à une tension de $30 \mathrm{kV}$ (maximum de courant $=48 \mathrm{kA}$ ). La gamme spectrale observée s'étend de 10 à $200 \mathrm{~nm}$.

L'émission présente un profil d'assez bonne symétrie circulaire, tout d'abord assez homogène (photo 1) avec une couronne qui s'accentue au cours du temps (photo 2) puis disparaît quelques dizaines
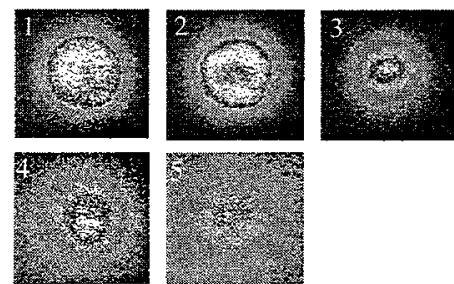

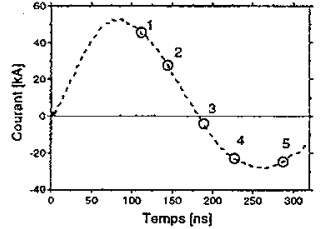
de ns plus tard (photo 3). La zone d'émission se concentre alors le long de l'axe sur une zone beaucoup plus étroite puis disparaît aux instants ultérieurs (plasma plus froid).

Les mêmes clichés ont été réalisés en intercalant un filtre en polyamide de $0,1 \mu \mathrm{m}$ d'épaisseur, ne laissant passer que le rayonnement compris entre 10 et $20 \mathrm{~nm}$ (X mous), ce qui permet d'observer isolément les ions de plus forte charge. On observe alors le même type de profil aux mêmes instants, à l'exception de la couronne jui n'apparaît pas dans cette gamme spectrale. On peut en déduire que cette couronne est constituée majoritairement d'ions de charge intermédiaire, créés par l'ablation des parois.

On peut conclure d'après ces images qu'un effet Z-pinch n'est pas réalisé dans nos conditions de décharge. D'autre part les photos 1 et 2 montrent que le plasma reste en contact thermique avec les parois du capillaire, par le biais de la couronne, ce qui exclut la possibilité d'un refroidissement brutal.

\section{ETUDE EN FONCTION DE LA LONGUEUR}

Un gain sur les raies de Balmer $\alpha$ et $\beta$ a été mis en évidence lors d'expériences préalables sur le même système [2], pour une capacité toutefois plus faible $(72 \mathrm{nF})$, donc un courant moins fort mais plus rapide. En revanche ce résultat n'a pas été reproduit sur le système actuel. L'influence de la longueur du plasma sur le profil d'émission a donc été étudiée, en vue de vérifier la validité des mesures de gain.

Dans un premier temps la densité de puissance est maintenue constante, ce qui impose au courant de varier (l'inductance variant avec la longueur). Le profil d'émission montre alors des évolutions temporelles très différentes d'une longueur à l'autre. Dans un deuxième temps, l'inductance est donc maintenue; on observe alors des instabilités, matérialisées par des points chauds, hors de l'axe du capillaire pour les faibles longueurs, rendant le plasma inhomogène. Ces mesures permettent d'expliquer la difficulté rencontrée pour reproduire le gain dans nos conditions expérimentales. Elles permettent également de penser que la rapidité du courant injecté est un facteur important dans l'obtention du gain.

\section{CONCLUSIONS ET PERSPECTIVES}

Les expériences d'imagerie pinhole menées ici montrent que le système de décharge n'est pas optimisé pour l'obtention du schéma de pompage désiré. L'effet Z-pinch nécessite une quantité initiale de matériau issu de l'ablation plus importante, ce qui impose de modifier le système de préionisation. Par ailleurs, un montage à ligne Blumlein permettant d'obtenir un courant plus rapide est actuellement à l'étude.

Ce travail a reçu le soutien financier du réseau TMR FACADIX (contrat $n^{\circ}$ ERBFMRXCT 980186)

\section{Références}

[1] R. Dussart, D. Hong, S. Götze et al., J. Phys. D, à paraître.

[2] R. Dussart, W. Rosenfeld, N. Richard et al., "Ablative capillary discharges as table-top XUV sources", 6th International Conference on X-Ray Lasers, Kyoto 31 août-4 septembre 1998, Y. Kato, H. Takuma et H. Daido Eds. (Institute of Physics Publishing, Bristol \& Philadelphia, 1999) pp. 171-174 